\title{
Durkheim a-t-il visé dans le Mill ?
}

La critique durkheimienne de la prétention à l'universalité de l'économie politique à la lumière de l'œuvre de J. S. Mill Had Durkheim a point against Mill ? The Durkheimian criticism of political economy's pretension to universality in light of J.S. Mill's work

Philippe Gillig

\section{(2) OpenEdition}

Journals

Édition électronique

URL : http://journals.openedition.org/ress/3353

DOI : $10.4000 /$ ress.3353

ISBN : $1663-4446$

ISSN : 1663-4446

Éditeur

Librairie Droz

Édition imprimée

Date de publication : 15 novembre 2015

Pagination : 219-244

ISSN : 0048-8046

\section{Référence électronique}

Philippe Gillig, « Durkheim a-t-il visé dans le Mill ? », Revue européenne des sciences sociales [En ligne], 53-2 | 2015, mis en ligne le 15 novembre 2018, consulté le 10 décembre 2020. URL : http:// journals.openedition.org/ress/3353; DOI : https://doi.org/10.4000/ress.3353 


\title{
DURKHEIM A-T-IL VISÉ DANS LE MILL? \\ LA CRITIQUE DURKHEIMIENNE DE LA PRÉTENTION À L'UNIVERSALITÉ DE L'ÉCONOMIE POLITIQUE À LA LUMIĖRE DE L'GEUVRE DE J. S. MILL
}

PHILIPPE GILLIG

Université de Strasbourg, BETA

phailaippe@yahoo.fr

Résumé. Émile Durkheim a noué un rapport critique aux économistes de son époque, dénonçant en particulier une supposée prétention à établir des lois naturelles. Leur échec tiendrait à leur hypothèse centrale d'un homo œconomicus - théorisée par John Stuart Mill - qu'ils prendraient pour une vérité intemporelle. Cet article tente de montrer que cette critique est précisément infondée en regard de l'œuvre de Mill. $\mathrm{Ce}$ dernier revendique au contraire clairement la relativité des lois économiques. En outre, la critique durkheimienne, qui semble d'abord d'ordre épistémologique, est en fait intimement liée à une dénonciation d'ordre politique et morale du laissez-faire. Mais là encore, nos deux auteurs se rejoignent bien plus que ce qu'on aurait tendance à croire: Mill est loin d'être un apologiste aveugle du marché.

Mots-clés: Durkheim, épistémologie, homo œeconomicus, laissez-faire, lois naturelles, Mill.

\begin{abstract}
Among all his criticisms towards economists, Émile Durkheim denounces in particular their alleged pretension to establish natural laws. In his view their failure stems from their central assumption of a homo œconomicus - theorised by John Stuart Mill — which they take for a timeless truth. In this article I argue that this criticism is unfounded in the case of Mill. The latter clearly asserts the relativity of economic laws. The Durkheimian criticism, which is primarily an epistemological one, involves also intimately a political and a moral attack against laissez-faire. But once again, the two authors have a great deal more in common than is frequently realized: Mill is far from being a blind advocate of the market.
\end{abstract}

Keywords: Durkheim, epistemology, economic man, laissez-faire, Mill, natural laws. 


\section{INTRODUCTION}

Parmi les nombreuses critiques adressées à l'économie politique, on trouve en particulier la dénonciation maintes fois répétée d'une certaine prétention des économistes à établir des lois universelles, à les présenter comme «naturelles», nécessaires ou immuables. Karl Marx a ainsi dénoncé la «naturalité » de l'économie politique, c'est-à-dire le fait que la théorie économique supposerait la propriété privée comme un fait «éternel» (Marx et Engels, I972 [I844], p. 32) et qu’elle considérerait par la même le régime capitaliste comme «la forme absolue et définitive de la production sociale » (Marx, I962 [1867], p. 19-20, postface de la seconde édition allemande du Capital, traduit par nous). Friedrich List a remis en cause le «cosmopolitisme» (Kosmopolitismus) de l'«école» d’Adam Smith, c'est-à-dire sa prétention à établir des vérités qui concernent le genre humain (List, I910 [1841], p. 20). Bruno Hildebrand affirme pour sa part que les mercantilistes, les physiocrates puis surtout Smith «et ses épigones» (il cite en particulier Rossi et Quincey) cherchent tous à établir des «lois qui devaient avoir une validité absolue en tout temps et pour tous les peuples» (deren Gesetze für alle Zeiten und Völker absolute Gültigkeit haben sollten, Hildebrand, I922 [I848], p. 2I). En France, Émile Durkheim reprend, à la suite d'Auguste Comte, cette tradition critique et prolonge en particulier les thèses de l'école historique allemande qu'il connaît relativement bien (Steiner, 2005, chap. I). Si Smith est l'épouvantail de List et Hildebrand, c'est John Stuart Mill qui se trouve principalement être dans le viseur de Durkheim.

Or, la critique durkheimienne ne nous semble pas véritablement fondée en regard des textes milliens. Il nous semble que l'interprétation de son œuvre fait l'objet, sur ce point précis de la critique, d’un contre-sens, ou à tout le moins d'une incompréhension. Durkheim critique en particulier la prétention des économistes à poser comme universelle une conception étriquée de l'être humain, le fameux homo oeconomicus théorisé justement par J.S. Mill à travers son concept de «désir de richesse» (Mill, I967 [1836]; 1974 [1843], liv. VI;

I Le fait que Smith ne focalise plus la critique chez Durkheim est sans doute à relier à l'influence de Comte qui, dans sa charge contre les économistes, épargnait déjà l'auteur de la Richesse des Nations (Comte, 1869 [1839], p. 195-198). 
I965a [1848], liv. II, iv). Mais cette accusation semble mal venue dès lors qu'on sait que Mill concevait les lois économiques non comme universelles mais comme relatives aux sociétés capitalistes modernes, du fait même que ces lois sont basées sur l'hypothèse du «désir de richesse». L’objet de cet article est donc précisément de revenir sur cet aspect de la critique durkheimienne en la confrontant aux écrits de J. S. Mill afin d’en évaluer la pertinence.

Dans cette perspective, nous tenterons dans un premier temps de mettre au clair la position durkheimienne, très spécifique, sur la question de la «naturalité » des lois économiques. Nous mettrons en évidence le fait que sa critique épistémologique est intimement liée à une dénonciation du «laissez-faire» (section I). À partir de là, nous montrerons que Durkheim néglige le souci dont fait montre Mill pour souligner la relativité des théories aussi bien que des systèmes économiques. De sorte que tant du point de vue de leurs principes épistémologiques que de leurs prises de positions d'ordre politico-moral, ces deux auteurs se rejoignent bien plus qu'ils ne s'opposent, contrairement à ce que Durkheim suggère (section 2).

\section{LA CRITIQUE DURKHEIMIENNE DE LA PRÉTENTION À L'UNIVERSALITÉ DU DISCOURS ÉCONOMIQUE}

Durkheim fait partie des auteurs qui, dans le sillage de Comte, ont vigoureusement critiqué l'économie politique classique. Il reproche aux économistes qu'il nomme «orthodoxes» à la fois leur prétention à l'indépendance vis-à-vis des autres sciences sociales, leur réduction de la société à une simple juxtaposition d'individus, et leur recours à l'abstraction (c'est-à-dire à la méthode hypothéticodéductive)². Or toutes ces critiques durkheimiennes tournent autour de la prétention des économistes à considérer «l'homme économique» comme l’archétype universel de tout être humain. Ainsi, à partir de la critique de la célèbre figure de l'homo œeconomicus, Durkheim va tenter de remettre en cause l'idée de «loi naturelle» des économistes «orthodoxes». Cette critique va être en réalité menée sur trois fronts : certes d’abord méthodologique, mais aussi politique et moral.

2 Pour une présentation et une discussion de ces critiques voir Phelep, 1984, chap. II ; et Steiner, 1994, p. 147-150 et 2005, p. 33-48. 


\section{I.I. LE RAPPORT AMBIGU DE DURKHEIM AUX «LOIS NATURELLES» DE L'ÉCONOMIE CLASSIQUE}

Il importe pour commencer de clarifier la position, originale et presque étonnante au premier abord, de Durkheim vis-à-vis de l'idée de «lois naturelles » en économie. Contrairement à d'autres auteurs critiques de la théorie économique comme Marx ou Veblen³ , le fait que l'économie politique prétende énoncer des «lois naturelles» ne lui pose pas, en soi, problème. On verra que c'est le type de lois qu'ils élaborent qui va constituer une pierre d'achoppement et que cela provient directement de la méthode avec laquelle ils les établissent.

\section{I.I.I. La revendication durkheimienne de l'existence de «lois naturelles » dans le monde social: l'éloge des économistes}

Selon Durkheim, on ne saurait reprocher aux économistes d’avoir naturalisé les faits sociaux ou les institutions humaines. Au contraire, il les complimente dès sa «Leçon d’ouverture » à son «Cours de sciences sociales 》 (I888) pour avoir été les premiers à reconnaître que «les lois sociales sont aussi nécessaires que les lois physiques » (Durkheim, 1987 [I888], p. 80). Durkheim illustre son assertion par deux lois classiques (la loi du prix unique en situation de concurrence et le fait que les prix s'accroissent avec la hausse de la population). Faisant remarquer que, dans le domaine politique, les économistes prônent en toute cohérence le «laissez-faire», il va même jusqu'à ajouter : «Étendez ce principe [de non-interventionnisme] à tous les faits sociaux et la sociologie est fondée» (ibid., p. 8I).

Rejetant la position des historiens qui dénient toute existence à de quelconques lois dans le monde social, il loue «le grand service que les économistes ont rendu aux études sociales» (ibid., p. 83-84) pour avoir compris que la société ne peut être instituée par artifice, du fait que «la vie collective est spontanée $»^{4}$ (ibid., p. 84). Cinq ans plus tard, sa célèbre thèse (De la division du travail social, I893) réexpose à l'identique cette idée :

3 Voir Veblen, 1919 (1898), p.61 et p.64-65.

4 Voir aussi Durkheim, 1887, p. 281. On ne manquera pas de relever la proximité théorique surprenante de ces analyses avec le concept hayékien d'« ordre spontané » (Hayek, 1952). Sur la proximité entre Hayek et Durkheim, voir Ege et Birner, 1999. 
C'est aux économistes que revient le mérite d'avoir les premiers signalé le caractère spontané de la vie sociale, d’avoir montré que la contrainte ne peut que la faire dévier de sa direction naturelle et que, normalement, elle résulte, non d’arrangements extérieurs et imposés, mais d’une libre élaboration interne. À ce titre, ils ont rendu un important service à la science de la morale (Durkheim, I994 [1893], p. 380).

Dans Les Règles de la méthode sociologique (1997 [1894]), il réaffirme l’idée de naturalité du social (mais sans évoquer cette fois une quelconque dette de la sociologie envers les économistes ${ }^{5}$. Au chapitre 2 de son magnus opus méthodologique il souligne le fait que les «praenotiones» nous font prendre la réalité sociale pour contingente, alors qu’en réalité elle est traversée par la nécessité. L'auteur met en garde contre l'illusion consistant à postuler le caractère «artificiel », « construit », « conventionnel » du social :

Même les phénomènes qui paraissent le plus consister en arrangements artificiels doivent être considérés [comme des choses, non comme des idées]. Le caractère conventionnel d'une pratique ou d'une institution ne doit jamais être présumé (ibid., p. 28, en italique dans le texte).

De même, dans un article publié en 1900 (et republié quasiment verbatim en 1915) intitulé «La sociologie en France au XIX siècle», il reconnaît sa dette intellectuelle envers les doctrines qui, de Condorcet à Espinas en passant entre autres par Comte et les économistes, posent que «les phénomènes sociaux sont naturels, c'est-à-dire rationnels, comme les autres faits de l'univers » (Durkheim, 1987 [1900], p. I30). Il y blâme alors la «conception artificialiste » de la société (ibid., p. I23). Cette dernière conception, un article de I887 («La science positive de la morale en Allemagne») l'associait déjà à Tarde en sociologie, Rousseau en philosophie, mais aussi en économie à certains représentants de la deuxième école historique allemande (Gustav Schmoller, Adolf Wagner) qui croient que les phénomènes moraux pourraient «être transformés à volonté par le législateur» (Durkheim, 1975 [1887], p. 280; voir aussi p. 283-284). On notera

5 Durkheim réaffirmera cependant en 1909 l'apport positif des économistes sur cette question dans un article intitulé «Sociologie et sciences sociales » (1987 [1909], p. 139). On notera qu'il rend hommage alors aussi à ce précurseur qu'est Condorcet, lequel est précisément l'autorité que Mill cite en épigraphe de son System of Logic (1974 [1843]). 
donc que les économistes «orthodoxes » ne sont, quant à eux, pas tombés dans ce que notre auteur voit comme un travers intellectuel.

Pour comprendre ce point de vue en faveur de l'existence de lois naturelles dans le monde social, il faut rappeler que Durkheim, à la suite de Comte, défend l'idée que la société est un «organisme » qui a sa vie intérieure propre et dont le fonctionnement obéit à des constances, des régularités, comme l'ensemble du règne naturel (Durkheim, 1987 [1900], p. I23). Ces régularités sont évidemment celles mises en évidence dans Le Suicide (1897). Elles désignent également, par exemple dans De la division du travail social, les règles sociales elles-mêmes, lesquelles sont la «forme définie que prennent avec le temps les rapports qui s'établissent spontanément entre les fonctions sociales» (Durkheim, I994 [I893], p. 360). Cette spontanéité des règles sociales, Durkheim la conçoit ainsi comme issue d'un processus en trois étapes de sédimentation de certaines actions sélectionnées au cours du temps : certaines «manières de réagir» au sein d’une société se répètent, donnant naissance à des «habitudes » et, in fine, à des «règles de conduite » (voir aussi Durkheim, 1975 [1887], p. 275 ; et 1994 [1893], p. 357).

Le naturel est donc inscrit au cœur du social pour Durkheim, et d’après lui, c'est tout l'apport de Comte que d’avoir montré que «les sociétés cessaient d’apparaître comme une sorte de matière indéfiniment malléable et plastique, que les hommes peuvent, pour ainsi dire, pétrir à volonté» (Durkheim, 1987 [1909], p. I4I)

\section{I.I.2. La compréhension positiviste par Durkheim des «lois naturelles» contre la conception des économistes}

Durkheim va même plus loin dans son apologie de la naturalité et considère que les économistes n’ont pas su naturaliser suffisamment les faits économiques: l'économie politique est restée «métaphysique », incapable d'établir des lois car «il lui manquait toujours une nature à observer» (Durkheim, 1987 [1888], p. 85). En réalité, lorsqu'il dit cela, il a en vue une acception très particulière du terme de «lois naturelles ». Il reproche en fait à les grands hommes «can do with society what they please » (1985 [I865], p. 322). 
l'économie classique son incapacité à établir des vérités conformes à ce qu'il regarde comme les canons de l'exigence scientifique moderne tels qu'Auguste Comte les a posés dans son Cours de philosophie positive (I830-I842), c'est-à-dire par induction. Les lois naturelles des économistes ne sont pas des «rapports de faits, objectivement observables entre les choses» mais des «connexions purement logiques entre des concepts formés d'une manière tout idéologique » (Durkheim et Fauconnet, 1975 [1903], p. I25) :

Pour l'économiste, il s'agissait, non de découvrir ce qui se passe dans la réalité, non de chercher comment des effets donnés y dérivent de causes également données, mais de combiner mentalement des notions toutes formelles, comme celle de valeur, d'utilité, de rareté, d'offre, de demande, etc. (ibid.).

Leurs lois ne seraient ainsi que des conséquences déduites de leur définition de l'individu, de simples implications logiques, et à ce titre les économistes baigneraient toujours dans «l'âgemétaphysique» défini par Comte (Durkheim, 1987 [1888], p. 85). Se retrouvant ainsi éloignés de l'expérience, de l'observation, en faveur de l'abstraction déductive, ils prennent (à tort) pour «naturel » ce qui n'est que «rationnel» (ibid.), là où le «naturel» devrait au contraire désigner l'empirique, ou plus précisément l'inductif, le positif. Dans Les Règles..., Durkheim renvoie explicitement à Mill (en fait au System of Logic) afin d'illustrer cette approche «idéologique». Affirmant que le «désir de richesse» est une «pure conception de l'esprit» (Durkheim, 1997 [1894], p. 24) qui doit d’abord être prouvée par l'expérience ou l'observation, il rejette catégoriquement sa méthode déductive.

En outre, cette méthode déductive a pour corollaire dommageable que les économistes (tout comme les moralistes) confondent la «science» (qui explique ce qui est) avec l'«art» (qui indique ce qui doit être) $)^{7}$. En effet, leurs prétendues «lois», en sus d'être peu nombreuses, consisteraient le plus souvent

7 La référence à Hume et à sa distinction entre «is 》 et «ought» est évidente (voir Hume, 1826 [1739], liv. III, part. I, sect. I); elle n'est toutefois pas mentionnée par Durkheim. On ne peut qu'être étonné de ce que Durkheim critique l'économie classique pour sa confusion entre «art » et «science » sans faire justement au moins une exception concernant Mill. Car comme le rappelle Swedberg (1994 [1987], p.56), c'est Mill qui le premier introduisit cette distinction en économie en Grande-Bretagne (Mill, 1967 [1836], p.33I et p.338; 1974 [1843], liv. VI, xii) avant d'être reprise par la plupart des économistes postérieurs à lui. 
en des «préceptes pratiques déguisés » (ibid., p. 26). Par exemple, la loi de l’offre et de la demande, qui n'est pas établie inductivement, relèverait de l'art, dans la mesure où elle ne fait que montrer qu'il est logique de suivre son désir de richesse (par exemple, vendre au prix le plus haut) si on entend bien son intérêt $^{8}$. Durkheim - qui semble surtout livrer ici sa compréhension toute personnelle de la loi de l'offre et de la demande - commente alors en ces termes :

Mais cette nécessité toute logique ne ressemble en rien à celle que présentent les vraies lois de la nature. Celles-ci expriment les rapports suivant lesquels les faits s'enchâ̂nent réellement, non la manière dont il est bon qu'ils s'enchaînent (ibid., p. 27, n. s.).

Durkheim, qui revendique ici la prétention à la «vraie naturalité » - c'est-àdire pour lui les lois naturelles obtenues par induction -, ajoute que sa remarque vaut pour toutes les lois économiques de «l'école orthodoxe». Ces dernières «sont naturelles [...] en ce sens qu'elles énoncent les moyens qu’il est bon ou qu’il peut paraitre naturel d’employer pour atteindre telle fin supposée» (ibid.). Mais elles ne sont pas naturelles au sens où elles renverraient à la «manière d'être de la nature, inductivement constatée » (ibid.). Dans «Sociologie et sciences sociales» (1909), il réaffirme ce point de vue : les économistes (comme les philosophes, à nouveau) entendraient les lois naturelles d'une façon particulière, à savoir comme ce que l'homme devrait suivre pour être en accord avec sa nature ; et Durkheim de remobiliser à nouveau la distinction art-science afin de distinguer l'économie (et la philosophie) d'une part - qui s'obstinerait à expliquer ce que les sociétés doivent être -, de la sociologie, d'autre part - laquelle montrerait les sociétés telles qu'elles sont (Durkheim, 1987 [1909], p. I39 et p. 151). Dans cet article de I909, Mill n’est pas nommément cité mais il n’est pas non plus épargné.

Ainsi, jusqu'ici la critique durkheimienne se situe à un niveau strictement méthodologique et semble plutôt claire: il s'agit de rejeter la déduction au profit de l'induction. Mais Durkheim brouille aussi quelque peu les pistes en détournant, voire en renversant, le sens du mot «naturel». Celui-ci ne renvoie pas à «immuable» ou à «éternel», au sens d'indépendant de toutes conditions historiques et empiriques, comme cela est le plus souvent d'usage dans 
le contexte de la dénonciation des lois «naturelles» (voir par exemple Marx, I96I [1857], p.6I8-6i9, ou I962 [1867], p. 95, note 32). Il renvoie au contraire, toujours dans le sillage de Comte, à l'idée de «nature empirique » et, partant, à la «diversité » de cette même nature. On comprend alors que Durkheim va pouvoir simultanément faire l’apologie de la naturalité du social tout en critiquant la prétention à une certaine universalité du discours économique qui justement négligerait la diversité empirique des faits économiques.

\section{I.2. LA CRITIQUE DE L'UNIVERSALITÉ DE L'HOMO ECONOMICUS ET DES LOIS BÂTIES SUR LUI}

Durkheim, dans les mêmes textes que ceux précités, n’en reste pas à un simple éloge des mérites naturalisants de l'école classique. Il insiste parallèlement, et avec au moins autant de force, sur la nécessité de relativiser leurs théories. Dans son «Cours de sciences sociales», Durkheim remet en cause la conception de la société, retenue par les économistes, comme simple juxtaposition d'individus. Surtout, il est intéressant pour nous de remarquer qu'il poursuit sa critique en leur reprochant en outre une définition appauvrie de l'individu:

Non seulement ils ont fait abstraction de toutes les circonstances de temps, de lieu, de pays pour imaginer le type abstrait de l'homme en général, mais dans ce type idéal luimême ils ont négligé tout ce qui ne se rapportait pas à la vie strictement individuelle si bien que d'abstractions en abstractions il ne leur est plus resté en main que le triste portrait de l'égoïste en soi (Durkheim, 1987 [1888], p. 85, n. s.) 9 .

Selon Durkheim, le recours à l'abstraction s'accompagne donc, chez les économistes, d'une conception simpliste de l'homme qu'ils prendraient pourtant comme une donnée anthropologique universelle. Dans «La sociologie et son domaine scientifique » (I900) il affirme de même :

Regardez les lois de l'économie politique ou, pour être plus exact, les propositions que les économistes parent de cette dignité! Indépendantes dans le temps et l'espace, elles ne semblent être solidaires d'aucune forme d'organisation sociale (Durkheim, 1975 [1900], p. 32, n. s.). 
En 1903, Durkheim loue par contraste ce qu'il voit comme une transformation récente de l'économie politique en Allemagne dans le sens d'un éloignement de ce caractère «idéologique», de sorte que l'économie ne s'oppose plus désormais à la sociologie. List en particulier, en posant qu'entre l'humanité et l'individu il y a la Nation, fait ressortir à juste titre que les «lois économiques varient d'un peuple à l'autre» (Durkheim et Fauconnet, 1975 [1903], p. I48), là où les classiques évoluent dans le Güterwelt, ce «monde isolé, partout identique à lui-même, et dans lequel le conflit de forces se résoudrait suivant des lois économiques inéluctables» (ibid., n. s.). Durkheim défend donc l'idée que la prise en compte de l'histoire par les auteurs allemands donne des «arguments contre les théories universalistes de l'École classique» (ibid., p. I49) et de citer List, Roscher - sans toutefois renvoyer à une seule analyse précise qui permettrait d’appréhender la teneur de ces «arguments».

Finalement, ce n’est que grâce aux éclaircissements précédents (voir section I.I.2) sur sa conception des lois naturelles du social, qu'on peut comprendre qu'il critique les lois immuables de l'économie classique et que simultanément il défend l'existence de lois naturelles dans le monde social. En effet, si la science sociale a bien pour but, d’après lui, de trouver des «invariants» (ibid.), cela doit être fait en usant de la méthode inductive, non de la déduction. Aussi, c'est l'approche d'un Gustav von Schmoller ou d'un Karl Bücher qu'il voit comme pertinente car ces derniers utilisent l'histoire comparative, étudiant des «faits éloignés dans l'espace et dans le temps » (ibid.). Là encore néanmoins, Durkheim n'illustre pas concrètement la fécondité de l'approche de ces auteurs et se contente de simples affirmations qu'il ne paraît pas excessif de qualifier de gratuites.

Si l'argumentaire durkheimien se situe avant tout sur un plan épistémologique, une lecture attentive révèle en fait que ses propos visent peutêtre plus fondamentalement des considérations d'ordre politique et moral, au-delà des simples observations de méthode. Cela se voit notamment dans «La science positive de la morale en Allemagne » (1887) où l'enjeu de la critique de la prétention à l'universalité de la théorie classique consiste en fait plus clairement à dénoncer l’absence de rôle économique que les économistes accordent aux pouvoirs publics. Tandis qu'il affirme que, «pour l'école de Manchester les lois économiques ne sont pas moins naturelles que celles 
de la pesanteur et de l'électricité, et par conséquent sont immuables... » (Durkheim, I975 [1887], p. 280) ${ }^{10}$, il sait gré à l'école historique allemande de faire rempart contre le «fatalisme économique» (Durkheim et Fauconnet, I975 [1903], p. 280). Plus précisément encore, dans son article de 1903, il soutient que la conception allemande a pour conséquence pratique de rendre concevable la possibilité pour l’État d'«agi[r] sur la conduite économique des individus» (ibid., p. I48). Ainsi, les Kathedersozialisten ont compris que «ce qui est réel, c'est la Volkswirtschaft, l’activité économique de la société qui a ses fins propres en matière économique » (ibid.). Dans un article du même nom, publié six ans plus tard (mais sans Fauconnet) et qui prolonge celui de 1903, on comprend clairement que cet intérêt porté par Durkheim aux «socialistes de la chaire » consiste en ce qu'ils réhabilitent la politique économique contre les économistes, pour lesquels la véritable organisation économique par l'État n'a jamais pu exister «car les hommes, sous l'influence de leurs gouvernants et par suite d'un véritable aveuglement, s'en seraient toujours laissé détourner»(Durkheim, 1987 [1909], p. I40). Durkheim prolonge en fait ici une critique qui était déjà présente chez $\operatorname{Comte}^{\mathrm{II}}$ : l'économie est vue comme la science des libéraux, ces chantres de la liberté individuelle qui s'échineraient à préserver cette liberté de toute intervention étatique. Or, si on se rappelle qu'un des thèmes centraux de la sociologie économique de Durkheim est le problème de la dérégulation (l'«anomie») de la production liée à l'accroissement de la division du travail, on comprend, comme le souligne Richard Swedberg, qu'«une grande partie de la véhémence des critiques que Durkheim adresse aux économistes vient de ce que ces derniers voient dans le laissez-faire un moyen de résoudre tous les maux de l'économie, alors que pour lui au contraire il est simplement synonyme d'une dérégulation plus importante » (Swedberg, 1994 [1987], p. 74).

I0 Le lecteur ne sait toutefois pas qui il inclut précisément dans «l'école de Manchester» ni d'ailleurs parmi les «orthodoxes».

II Dans la 47e leçon du Cours de philosophie positive, Comte vitupère contre le «stérile aphorisme de liberté industrielle absolue» (I869 [1839], p. 202), ce «dogme universel [de] l'absence nécessaire de toute intervention régulatrice» (ibid., p. 200). 
Durkheim opère un second déplacement de la critique, sur le terrain de la morale et de la justice cette fois. On sait que le sociologue français a toujours insisté sur l'idée que les faits sociaux sont des faits moraux, au sens où ils se présentent à l'individu sous la forme d'une obligation qui s'impose à lui. Mais la morale ne doit précisément pas être entendue au sens des économistes partisans de «l'utilitarisme anglais » qui définissent la morale par l'utile (Durkheim, I975 [I887], p. 268). Si pour Durkheim les faits sociaux que sont les faits économiques sont des faits moraux, c'est qu'ils sont indissolublement liés à la question de la justice, non à l'utile comme le souligne Steiner (2005, p. 4I-42 ; p. 48-57). Dans la De la division... en particulier, les lois naturelles des économistes entrent en opposition la plus patente avec la question de la justice. Durkheim considère que, pour que la division du travail génère de la solidarité sociale, il faut que chaque individu consente à sa tâche. Il faut par conséquent une distribution des fonctions sociales en adéquation avec la distribution des talents naturels. Or, cela suppose que rien n'entrave certains concurrents à la faveur d'autres, ou comme le formule Durkheim, cela suppose «l'absolue égalité dans les conditions extérieures de la lutte» (Durkheim, I994 [1893], p. 37I). Ce qui permet de réaliser cette justice dans l'échange et le contrat c'est la suppression - ou du moins la limitation drastique - de l'héritage : «...il ne peut pas y avoir des riches et des pauvres de naissance sans qu'il y ait des contrats injustes » (ibid., p. 378). C'est donc à la condition sine qua none de mettre un terme à la transmission héréditaire des patrimoines que la division du travail sera «spontanée », c'est-à-dire qu’elle permettra «le libre déploiement de la force sociale que chacun porte en soi» (ibid., p. 370).

Or, c'est là que Durkheim mobilise le même argumentaire que celui déployé ailleurs sur le plan méthodologique (voir supra) : les économistes ont certes signalé en premier le «caractère spontané de la vie sociale» mais ils «se sont mépris sur la nature de cette liberté » (ibid., p. 380), en en faisant un attribut constitutif de l'homme, déduit logiquement du concept de l'individu en soi, et qui existerait dès l'état de nature. Dit autrement, les économistes se trompent car, en prenant la liberté pour l'absence de toute réglementation, ils adoptent un concept universel de liberté tiré de l'état de nature. Contre 
cet universalisme, Durkheim défend, quant à lui, l'idée que non seulement «la liberté elle-même est le produit d'une réglementation » (ibid., p. 380), mais que chaque type de société exige une réglementation qui corresponde à la «nature vraie des choses » (ibid., p. 370), c'est-à-dire propre à la nature particulière de la solidarité qui la caractérise. Par exemple, dans une société de castes, l'héritage ne représente pas une contrainte injuste, tandis que, dans une société où règne la division du travail, l'héritage contrevient à la justice et est source de division du travail «contrainte», autre forme anormale de la division du travail (à côté de la forme «anomique»). De sorte que, dans le cas d'une société fondée sur la division du travail, la prescription universelle des économistes d'une absence de réglementation dans le domaine des affaires économiques (en particulier leur refus d'une réglementation de l'héritage), parce qu'elle néglige la diversité des types de société, génère de la souffrance et menace la condition même de possibilité d'existence d'une telle société. Nous verrons (dans la section 2) que Mill défend en réalité des positions extrêmement proches de celles de Durkheim.

Durkheim rappelle enfin que c'est la «Volkswirtschaft » au sens de Schmoller et Wagner, qui est réelle, cet être social qui «institue et organise, pour les satisfaire, une activité économique qui [est celle] de la nation dans son ensemble», de sorte que les agents privés (Einzelwirtschaften) ne constituent en réalité qu’une abstraction fallacieuse (Durkheim, I975 [1887], p. 273). Durkheim demande alors de façon rhétorique : «Est-ce que les utilitaires n’ont pas, eux aussi, fait de l'intérêt collectif la base de la morale? Oui, mais pour eux cet intérêt collectif n'est qu'une forme de l'intérêt personnel». Or, «[1]a science économique [doit] se préoccupe[r] d’abord des intérêts sociaux et par contrecoup seulement des intérêts individuels » (ibid.). Ainsi se trouve connectée l'épistémologie à la morale utilitariste : la prétention universalisante des modèles abstraits découle d’une conception erronée de la morale comme simple égoïsme. 


\section{I.3. UNE CRITIQUE QUI SE TIENT À UN NIVEAU TROP GÉNÉRAL}

Avant d'examiner la position de Mill sur ces questions, on retiendra que la charge durkheimienne à l'encontre d'une universalité des lois économiques classiques n'est pas vraiment convaincante, dans la mesure où notre auteur se contente de commenter assez superficiellement l'œuvre économique des uns et des autres sans jamais prendre d'exemple précis. La principale (et presque seule) loi économique qu'il discute - à notre connaissance - est celle de l'offre et de la demande (voir supra i.I.2.), mais sans que le lecteur sache précisément ce qu'il entende par là. Durkheim est d'ailleurs loin de faire montre de la même maîtrise du corpus classique qu'un Marx par exemple. Phelep rappelle que Durkheim (tout comme Comte d'ailleurs) n'avait qu'une connaissance partielle et superficielle des classiques (Phelep, 1984, p. 213, note 2). Guy Aimard affirme à ce sujet que «bien qu'il soit pratiquement impossible de déterminer quels furent les ouvrages d'économie politique composant sa bibliothèque, celle-ci ayant été dispersée sous l'occupation, on peut augurer qu'il eut une bonne connaissance des grands classiques - Smith, Ricardo, Say - lors de la préparation de sa thèse sur la division du travail social» (Aimard, 1962, p. 5-6). On sait qu'il a lu et travaillé sur Sismondi (Lacroix et Landerer, 1972, p. 162). Steiner rappelle, après une analyse exhaustive des références aux économistes dans l'œuvre de Durkheim, que son rapport aux économistes s'est traduit d'abord par un certain intérêt puis par un éloignement progressif, surtout après I895-1896, dû à son insatisfaction à leur égard (Steiner, 2005, p. 21-22). Durkheim, non seulement les cite alors de moins en moins mais surtout de plus en plus à travers une appellation générique («les économistes», «économisme», «science économique», «économie politique»). L'utile travail de Steiner permet aussi de faire ressortir que les références aux classiques sont numériquement minces et en outre essentiellement allusives (Steiner, 2005, p. 24).

Pour ce qui concerne plus spécifiquement Mill, ce dernier n’est cité que deux fois et au sujet de considérations d'ordre très général dans De la division... Mill est d'abord évoqué pour être rangé avec Smith parmi ceux qui auraient espéré que la division du travail n’affecte pas l'agriculture (Durkheim, I994 [I893], p. 2). 
Au chapitre IV du livre II ensuite, Durkheim cite rapidement les Principles de Mill, lesquels véhiculeraient l'idée commune que «la principale raison d'être [de la division du travail] serait "de classer les individus suivant leurs capacités" " (ibid., p. 29i). On notera au passage que la référence, en note de bas de page, associée à cette citation, est une des rares de l'ouvrage à ne pas renvoyer à une pagination précise, pas même à une section du livre, alors que Durkheim fait montre en général d’une grande précision dans son référencement bibliographique. Par ailleurs, nous ne savons pas quels écrits de Mill il aurait étudié, à part vraisemblablement le System of Logic. Le seul passage cité de cet ouvrage - dans Les Règles... (voir supra I.I.2.) - est extrêmement laconique ${ }^{12}$, et la référence en note de bas de page renvoie étrangement au livre III du System of Logic, alors que c'est au livre VI (ch. ix) que Mill expose la conception de la science économique à laquelle Durkheim fait référence, comme l’a aussi vu Steiner (2005, p. 38).

Il ressort ainsi que Durkheim, en usant des termes «les économistes», «les orthodoxes», «l'école de Manchester», etc., fait fonctionner des ensembles qui apparaissent bien vides. Comme en témoigne notre analyse de De la division..., il vise en réalité bien plus les libéraux que les classiques. Et avec Steiner, on peut dire que ce sont d'ailleurs essentiellement les libéraux français du Journal des économistes (Steiner, 2005, p. 25), lesquels sont regroupés au sein de la Société d'économie politique. Sa critique pèche donc par son caractère extrêmement généralisant, d’autant que celui-ci n’est pas compensé par la production d'écrits de sociologie économique dont on verrait concrètement la fécondité. En effet, comme le remarque Steiner, Durkheim n’a produit aucun écrit économique et «ne signe aucun compte rendu dans la cinquième section de l'Année sociologique consacrée à la sociologie économique » alors qu’il contribue à toutes les autres sections (Steiner, 2005, p. 21). Tout au plus se contente-t-il d'esquisser des pistes qu'il faudrait suivre en vue d'opérer un traitement positiviste des faits économiques.

12 Voici cette citation: «[L'économie politique] a pour objet, dit Stuart Mill, les faits sociaux qui se produisent principalement ou exclusivement en vue de l'acquisition des richesses» (Durkheim, 1997 [1894], p. 24). 


\section{LE CONTRESENS DE DURKHEIM SUR LE RAPPORT DE MILL À L'UNIVERSALITÉ DE LA THÉORIE ÉCONOMIQUE}

Dans ce discours généralisant sur les économistes, la figure de Mill ne peut qu' interroger. Car Durkheim opère, selon nous, un contresens sur la fonction même de l'abstraction au sein du cadre théorique millien. Comme nous l'avons souligné précédemment, Durkheim associe l'abstraction en économie à une prétention à l'universalité. Or Mill, au contraire, la conçoit comme une particularisation, ou dit autrement, comme une méthode qui limite la validité des théories économiques.

\section{I. LA CONCEPTION MILLIENNE DE L'ABSTRACTION}

En effet, dans ses deux grands écrits d'épistémologie économique - son Essay on the Definition of Political Economy (I836) et son System of Logic (I843) - ainsi que dans ses Principles (1848), Mill cherche clairement à limiter le domaine de validité de l'économie politique du fait même que cette science est fondée sur l'hypothèse du «desire of wealth». Rappelons d'abord que, dans un passage de son essai On the Definition of Political Economy (passage qui sera repris quasiment verbatim dans le System of Logic [Mill, I974 (I843), p. 90I-902]), Mill pose que :

[Political Economy] is concerned with [man] solely as a being who desires to possess wealth [...]. It predicts only such of the phenomena of the social state as take place in consequence of the pursuit of wealth. It makes entire abstraction of every other human passion or motive; except $[. .$.$] aversion to$ labour, and desire of the present enjoyment of costly indulgences (Mill, 1967 [1836], p. 32I-322).

Il ne fait néanmoins aucun doute qu'il regarde le «désir de richesse» comme une stricte hypothèse qui n’a pas vocation à décrire la réalité. Mill revendique sans ambiguïté l'idée que la science économique repose sur une vision réductrice de l'homme, qui fait de lui un être mû exclusivement par son intérêt égoïste. Mais il précise aussi d'emblée qu’il ne s'agit pas là d’une description réaliste de l'activité humaine mais d'une abstraction nécessaire pour permettre une étude scientifique de certains phénomènes : 
Not that any political economist was ever so absurd as to suppose that mankind are really thus constituted, but because this is the mode in which science must necessarily proceed. When an effect depends on a concurrence of causes, these causes must be studied one at a time, and their laws separately investigated, if we wish, through the causes, to obtain the power of either predicting or controlling the effect (Mill, I974 [1843], p. 902 citant son propre essai de I836).

Mill a conscience que l'action d'un individu s'explique par un entrelacs de causes extrêmement complexe (les traditions, les valeurs, etc.) qu'il regroupe sous le terme de «coutume» (custom) dans le Livre II, chap. iv des Principles ${ }^{13}$. Il se réclame d’ailleurs de Comte et de sa notion de «consensus » (une notion également prisée par Durkheim), c'est-à-dire d'interdépendance entre tous les phénomènes sociaux (voir le System of Logic, liv. VI, chap. ix-x, et Mill I985 [1865], p. 305). Mais, il maintient contre Comte - comme souligné précédemment - qu'une étude scientifique des phénomènes sociaux requiert de commencer par une étude séparée, c'est-à-dire de procéder par abstraction. L'économie politique est ainsi envisagée «as the necessary basis of the higher branches of the [social] science» (Mill, 1985 [1865], p. 306 note). Ce n'est donc pas par myopie que Mill opère cette réduction, mais uniquement par souci analytique. Mill conçoit l'économie politique comme une science «abstract or hypothetical» (Mill, 1965a [1848], p. 239), c'est-à-dire reposant sur l'isolement d’un comportement spécial : le désir de richesse. Par «hypothétique» il faut entendre que cette science détermine ce que seraient les phénomènes sociaux si cette cause spéciale agissait seule ; elle est donc «abstraite» dans la mesure où elle fait abstraction de toute «disturbing causes» (i.e. les motivations qui constituent la coutume) qui, dans la réalité, provoqueraient d’autres comportements. Les lois économiques n’ont donc rien d'«éternel»ou d'«atemporel» : elles sont au contraire des «tendances» (Mill, I974 [1843], p. 899 et p. 910) puisqu'elles indiquent ce qui se produirait si les individus n'étaient mus que par leur «desire of obtaining the greatest quantity of wealth with the least labour and self-denial » (ibid., p. 903).

13 Dans les Principles, Mill désigne plus précisément à travers ce terme un ensemble d'obligations tacites ou explicites qui contrarient le libre choix égoïste. 


\subsection{MILL ET LA QUESTION DU DEGRÉ DE VALIDITÉ DES LOIS ÉCONOMIQUES}

De là découle une extrême prudence chez Mill sur la question de l'applicabilité des résultats et des théorèmes économiques. Si l'on veut utiliser ces lois abstraites et conditionnelles dans la pratique, il faut encore rétablir tout ce qu'elles ont négligé dans leur hypothèse de départ (toutes les motivations ou les institutions sociales qui contrecarrent in concreto le désir de richesse) - ce qui implique dans chaque cas étudié une étape de vérification de la validité empirique de cette hypothèse (Mill, I974 [1843], p. 87I, p. 874 et p. 896-897). L'économie politique ne saurait servir de guide pratique dont on pourrait intemporellement tirer des préceptes. Plus précisément, Mill limite le domaine de validité de l'économie politique aux économies concurrentielles. En effet, la libre concurrence est le cadre juridique qui permet à cette motivation humaine qu’est le désir de richesse de s'épanouir pleinement. La concurrence constitue donc une hypothèse de base de l'économie politique, tout aussi indispensable que celle du «désir de richesse » puisqu'elle en est le complément :

Only through the principle of competition has political economy any pretension to the character of a science. So far as rents, profits, wages, prices, are determined by competition, laws may be assigned for them. [...] As an abstract or hypothetical science, political economy cannot be required to do, and indeed cannot do, anything more (Mill, 1965a [1848], p. 239).

Pour cette raison précise, Mill pose comme première limitation à la validité de l'économie politique que ses lois ne concernent qu'un nombre très restreint de pays, anglo-saxons en l'occurrence, dans lesquels «the produce is shared among three classes, altogether distinct from one another, labourers, capitalists, and landlords; and that all these are free agents, permitted in law and in fact to set upon their labour, their capital, and their land, whatever price they are able to get for it», ce qui constitue «a supposition which is scarcely realized anywhere out of England and Scotland» (Mill, I974 [I843], p. 903). En outre, la disposition égoïste et son pendant institutionnel qu'est la libre concurrence sont une caractéristique des sociétés anglo-saxonnes modernes uniquement : «Competition, in fact, has only become in any considerable degree the governing principle of contracts, at a comparatively modern period. The farther we look back into history, the more we see all transactions and engagements under the influence of fixed customs » (Mill, 1965a [1848], p. 240). 
Mill fait donc clairement apparaître la nature historiquement relative des conclusions de l'économie politique. En réponse aux attaques de Comte à l'encontre de l'économie politique, Mill affirme par conséquent quaucun économiste ne «pretend that the laws of wages, profits, values, prices, and the like, set down in their treatises, would be strictly true, or many of them true at all, in the savage state (for example)...» (1985 [1865], p. 305). Sur ce point, Mill a donc non seulement répondu par anticipation aux assauts de Durkheim, mais en outre, a procédé lui-même à une critique de l'économie politique, dénonçant les économistes qui oublient cette historicité : «the principal error of narrowness with which they are frequently chargeable, is that of regarding, not any economical doctrine, but their present experience of mankind, as of universal validity; mistaking temporary or local phases of human character for human nature itself» (ibid., p. 306).

Mill a donc parfaitement conscience de ce que les lois établies par les économistes sont relatives à ce système historique particulier qu'est le capitalisme marchand. Aussi, l'assertion durkheimienne précitée (section I.2) selon laquelle les lois déduites par les économistes de l'hypothèse d'un homo œconomicus seraient «indépendantes dans le temps et l'espace [et] semblent être solidaires d'aucune forme d'organisation sociale » ne rend pas justice à la pensée de Mill. Il nous est ainsi possible de conclure que la critique durkheimienne de la prétention à l'universalité de l'économie classique semble largement infondée en regard de l'œuvre particulière de Mill, en ce que cette critique passe à côté de la réflexion millienne sur l'applicabilité relative des théories économiques. Partant, nous ne pouvons que nous associer au jugement de Steiner lorsqu'il affirme que «la critique que Durkheim fait de Mill est injuste» (Steiner, 1994, p. I5 I), même si les raisons que nous avons avancées ici sont, tout en étant complémentaires, pour l'essentiel différentes des siennes.

\subsection{MILL ET LA QUESTION DU «LAISSEZ-FAIRE»}

Nous avons vu que, derrière l'appellation générique des «économistes » univoquement critique sous la plume de Durkheim, ce dernier semble fantasmer un ennemi théorique dont la dangerosité s'exerce, en dernière instance, sur le plan politico-moral: comme chez Comte, l'économie politique est perçue comme le cheval de Troie intellectuel du «laissez-faire», 
c'est-à-dire comme la justification idéologique d'un individualisme destructeur de toute cohésion sociale. Alors qu’on présente en général la critique des économistes par Durkheim comme essentiellement d'ordre méthodologique (Phelep, 1984; Steiner, 2005), on peut se demander si l'enjeu fondamental de cette critique ne se situe pas sur le plan politique : les économistes représentent un danger moral et politique en ce qu'ils prônent un individualisme «égoïste » ou «utilitaire ( (Durkheim, 1987 [I898], p. 262-263) incompatible avec la cohésion sociale. Pourtant là encore, Mill est un auteur à même de faire s'évanouir une bonne partie de tels fantasmes.

En effet, conformément à sa méthodologie relativiste, Mill considère que, du point de vue politique, l'économie concurrentielle capitaliste n'est qu'une simple institution possible. Rejetant tout fatalisme à son égard, il est resté célèbre, au sein du corpus classique, pour ses prises de position réformatrices, son «laissez-faire » très nuancé et pour s'être même déclaré «socialiste » dans son autobiographie (Mill, I98I [1873], p. 239)². Comme Smith, il a consacré une partie du cinquième livre de son magnum opus économique (intitulé On the Influence of Government) à préciser la légitimité de l'intervention de l’État dans la sphère marchande. Mill y dénonce les rentes indues (unearned fortunes ${ }^{15}$ ) et le salariat (wage system) qui génèrent un antagonisme de classe : «So long, however, as the great social evil exists of a non-labouring class, labourers also constitute a class, and may be spoken of, though only provisionally, in that character » (Mill, 1965 [I [848], p. 758 ; voir aussi, p. 766-767). Dès les deux premières éditions des Principles (1848 et I849), il suggère comme remède la participation des travailleurs aux bénéfices (Principles, IV, vii, p. 5). Mill s'est prononcé à partir de la $3^{\mathrm{e}}$ édition des Principles (1852), et avec de plus en plus de force, pour le développement des coopératives ouvrières de production, comme en témoignent les remaniements de ce chapitre vii du Livre IV (intitulé «On the Probable Futurity of the Labouring Classes») au fil des éditions successives des Principles.

14 Son «socialisme» a été l'objet de débat dans la littérature. Sur cette question on pourra consulter Feuer, 1949; Hollander, 1985, p.770-824; Robbins, 2004 [1998], p. 231 -237.

15 Voir Mill, 1965a (1848), p. 216; 1965b (1848), p.8II, p.819 et p.821. 
On peut même aller plus loin et dire qu'un des objectifs des Principles était, comme le souligne De Marchi, de «rescue from narrow, negative, and inflexible writers of the extreme laissez-faire persuasion "the truths they misapply, and [combine] these with other truths to which they are strangers...” $\rangle^{16}$ (Marchi, 1974, p. 136). Parmi les auteurs représentatifs de cette posture, on trouve Harriet Martineau, Edward Baines Jr. et Thomas Hodgskin. De Marchi rappelle que les deux derniers «backed this practice [laissez-faire] with the imperative of natural law (ibid.). Dans le cas de Martineau, on trouve trace chez Mill dès I833 (dans une lettre à Carlyle du I I-I2 avril) de cette dénonciation d'une conception étriquée du «laissez-faire » : «Your criticism on Miss Martineau is, I think, just : she reduces the laissez faire system to absurdity as far as the principle goes, by merely carrying it out to all its consequences » (Mill, 1963, p. 152).

Sur le plan de la morale, Mill développe une conception de la justice sociale fondée sur un idéal méritocratique utilitariste étonnamment proche de celui de Durkheim, malgré les dires de ce dernier :

It is not the fortunes which are earned, but those which are unearned, that it is for the public good to place under limitation. [The] impartiality [of a just and wise legislation] between competitors would consist in endeavouring that they should all start fair. [...] Many, indeed, fail with greater efforts than those with which others succeed, not from difference of merits, but difference of opportunities (Mill, 1965b [1848], p. 81 I, n. s.).

Pour Mill, les éventuelles inégalités de positions et de revenu peuvent être déclarées légitimes si et seulement si elles sont fondées sur le travail et le mérite. Or, pour que l'égalité des chances soit effective, il souligne qu'il faut tenter de neutraliser autant que faire se peut les inégalités liées à la naissance par une intervention publique à deux niveaux: l'éducation des masses via l'instruction publique gratuite (qui permettra en outre de limiter les naissances, principale cause du paupérisme) et la limitation de l'héritage par la mise en place d'un impôt progressif et confiscatoire au-delà d'un certain seuil (Mill, I965b [I848], p. 8II-8I2). Autant de propositions qui visent à accomplir l'idéal durkheimien d’une «égalité dans les conditions extérieures de la lutte» (voir supra I.2). Précisons qu’une telle intervention des pouvoirs publics 
est fondée «on grounds of general expediency (Mill, ibid., p. 8I I), c'est-à-dire sur une argumentation utilitariste au sens que Mill donne à ce terme dans son ouvrage éponyme : le principe d'utilité, ou principe du plus grand bonheur du plus grand nombre, a pour fondement «not the agent's own greatest happiness, but the greatest amount of happiness altogether» (Mill, 1985 [186I], p. 213). Le principe d'utilité n'est donc aucunement assimilable au «desire of wealth». L'utilitarisme de Mill n'étant pas fondé sur le «desire of wealth», il ne constitue aucunement la philosophie implicite de son économie politique; bien au contraire, elle en constitue plutôt le négatif.

Ainsi, l’association faite assez systématiquement par Durkheim entre «l'égoïsme» et la «morale utilitaire» des économistes (voir supra) relève également d'un contresens, en tout cas si l'on s'en tient à Mill. Sur le plan politique, cette défense de la méritocratie a conduit Mill à préconiser la confiscation par l'État de la rente foncière non méritée, c'est-à-dire celle due à la seule hausse de la population. Cette idée apparaît dès la première édition des Principles (V, ii, p. 5) puis sera reprise en 187I, dans son Explanatory Statement of the Programme of the Land Tenure Reform Association de 187I, association éphémère dont Mill fut président et qui visait une réforme de la propriété de la terre (Mill, I967 [1871], p. 687 et suiv.).

Ces déclarations franches en faveur d'une régulation du marché par l'État, lesquelles ne remettent aucunement en cause son économie politique, révèlent in fine une étonnante communauté de vue avec Durkheim sur le plan des idées politiques et de la justice sociale.

\section{CONCLUSION}

Il ressort que la critique méthodologique durkheimienne d'une prétention à l'universalité des classiques est très spécifique: Durkheim ne cherche pas à remettre en cause l'existence de lois naturelles en économie - dans la mesure où il croit lui-même à l'existence de lois sociales naturelles - mais à dénoncer les prétentions de la méthode hypothético-déductive, qui conduirait à ignorer la relativité socio-historique des lois bâties à partir d'elle. Cette critique méthodologique, qui emporte cet adversaire indistinct que sont «les économistes», s'avère en réalité peu pertinente. D’abord parce que, lorsqu’on concentre l'at- 
tention sur l'un de ces économistes visés - John Stuart Mill, dont le cas est exemplaire puisqu'il est à la fois le dernier grand représentant classique et l'économiste qui a proposé la définition la plus travaillée de l'objet de l'économie politique classique - les choses se révèlent plus complexes : Durkheim méconnaît les positions relativistes de Mill, lequel s'avère défendre in fine une position méthodologique beaucoup moins naturalisante que Durkheim. Le recours à cette abstraction qu'est l'homo œeconomicus est un pis-aller assumé pour Mill qui considère que les sciences sociales ne peuvent prétendre établir que des vérités relatives. En second lieu, la critique tourne tout aussi court du côté politico-moral: Mill a été une des figures de proue de l'économie politique classique tout en se faisant ouvertement l'avocat du socialisme. Et l'accusation de vouloir propager universellement un individualisme égoïste et utilitaire est d’autant plus infondée que Mill est précisément utilitariste.

Cela va sans dire, cette critique que nous adressons à Durkheim ne condamne aucunement l'ensemble de son œuvre. Son objectif n'était évidemment pas de rédiger une étude rigoureuse sur Mill, comme nous avons nousmême cherché à le faire en tant qu'historien de la pensée. Reste que les écrits de Durkheim présentent un caractère nettement polémique qui peut s'expliquer par des raisons essentiellement extra-théoriques. Il faut se souvenir que la sociologie naissante est alors en lutte pour une reconnaissance académique face à une science économique qui revendique une existence depuis au moins un siècle et qui est en sérieuse voie d'institutionnalisation. Karady rappelle à ce titre que les sociologues d'obédience durkheimienne furent largement exclus des facultés de droit - lesquelles hébergeaient alors les chaires d'économie - en dépit de leurs demandes répétées d’intégration (Karady, 1979, p. 58-59). Dans la perspective de la constitution de son école, Durkheim émet une charge qui est donc aussi largement stratégique, visant à fédérer en s'opposant ${ }^{17}$. 


\section{BIBLIOGRAPHIE}

AIMARD G., 1962, Durkheim et la science économique: l'apport de sa sociologie à la théorie économique moderne, Paris, PUF.

COMTE A., 1869 (1839), Cours de philosophie positive, Paris, Baillière et Fils.

DURKHEIM É., 1975 (1887), «La science positive de la morale en Allemagne», in Id., Textes, Paris, Éditions de Minuit, vol. I, p. 267-343.

-, 1975 (1900), «La sociologie et son domaine scientifique», in Id., Textes, Paris, Éditions de Minuit, vol. I, p. 13-36.

-, 1987 (1888), «Cours de sciences sociales, Leçon d'ouverture », in La Science sociale et l'Action, Paris, PUF, «Quadrige», p. 77-II0.

-, 1987 (1898), «L'individualisme et les intellectuels», in La Science sociale et l'Action, Paris, PUF, «Quadrige», p. 261-278.

-, 1987 (1900), «La sociologie en France au XIX siècle», in La Science sociale et I'Action, Paris, PUF, «Quadrige», p. III-136.

-, 1987 (1909), «Sociologie et sciences sociales», in La Science sociale et l'Action, Paris, PUF, «Quadrige», p. 137-159.

-, 1994 (I893), De la division du travail social, Paris, PUF, «Quadrige».

-, 1997 [1894], Les Règles de la méthode sociologique, Paris, PUF, «Quadrige».

- et FAUCONNET P., 1975 (1903), «Sociologie et sciences sociales», in Émile Durkheim, Textes, Paris, Éditions de Minuit, p. 121-159.

EGE R. et BIRNER J., 1999, «Hayek with Descartes and Durkheim: Reason and the Individual», The American Journal of Economics and Sociology, 58-4, p. 749-780.

FEUER L. S., 1949, «John Stuart Mill and Marxian Socialism», Journal of the History of Ideas, 10-2, p. 297-303.

HAYEK F. A., 1952, The Counter-Revolution of Science: Studies On the Abuse of Reason, Glencoe, Free Press.

HILDEBRAND B., 1922 (I848), Die Nationalökonomie der Gegenwart und Zukunft, und andere gesammelte Schriften, Hans Gehrig (dir.), Jena, Gustav Fischer.

HOLLANDER S., 1985, The Economics of John Stuart Mill, Toronto, University of Toronto press, «Studies in Classical Political Economy». 
HUME, D., 1826 (1739), A Treatise of Human Nature, The Philosophical Works of David Hume II. Edinburgh, A. Black \& W. Tait.

KARADY V., 1979, «Stratégies de réussite et modes de faire-valoir de la sociologie chez les durkheimiens», Revue française de sociologie, 20-I, p. 49-82.

LACROIX B. et LANDERER B., 1972, «Durkheim, Sismondi et les socialistes de la chaire», L'Année sociologique, 23, p. 159-204.

LIST F., 1910 (|84I), Das nationale System der politischen Ökonomie, Jena, Gustav Fischer.

MARCHI N. B. (de), 1974, «The Success of Mill's Principles», History of Political Economy, 6-2, p. II9-157.

MARX K., 196I (1857), Einleitung zur Kritik der politischen Ökonomie, Berlin, Dietz Verlag, Marx Engels Werke.

-, 1962 (1867), Das Kapital, Berlin, Dietz Verlag, Marx Engels Werke.

- et ENGELS F., 1972 (I844), Die heilige Familie oder Kritik der kritischen Kritik, Berlin, Dietz Verlag, Marx Engels Werke.

MILL J. S., 1963, The Earliers Letters of John Stuart Mill 1812-1848 Part I, in Id., vol. XII, The Collected Works, Toronto, University of Toronto Press.

-, 1965a (1848), The Principles of Political Economy with Some of Their Applications to Social Philosophy (Books I-II), in Id., The Collected Works, vol. II, Toronto, University of Toronto Press.

-, 1965b (1848), The Principles of Political Economy with Some of Their Applications to Social Philosophy (Books III-V and Appendices), in Id., The Collected Works, vol. Ill., Toronto, University of Toronto Press.

-, 1967 (1834), «Miss Martineau's Summary of Political Economy», in Id., The Collected Works, vol. IV, Toronto, University of Toronto Press, p. 223-228.

-, 1967 (1836), «On the Definition of Political Economy; and on the Method of Investigation Proper to It », in Id., The Collected Works, vol. IV, Toronto, University of Toronto Press, p. 309-339.

-, 1967 (I87I), «Land Tenure Reform», in Id., The Collected Works, vol. IV, Toronto, University of Toronto Press, p. 687-696.

-, 1972, The Latter Letters of John Stuart Mill 1849-1873 Part I, in Id., The Collected Works, vol. XIV, Toronto, University of Toronto Press. 
-, 1974 (1843), A System of Logic Ratiocinative and Inductive, Being a Connected View of the Principles of Evidence and the Methods of Scientific Investigation (Books IV-VI and Appendices), in Id., The Collected Works, vol. III, Toronto, University of Toronto Press.

-, I98I (1873), Autobiography, in Id., The Collected Works, vol. I, Toronto, University of Toronto Press.

-, 1985 (I86I), Utilitarianism, in Id., The Collected Works, vol.X, Toronto, University of Toronto Press, p. 203-260.

-, 1985 (I865), Auguste Comte and Positivism, in Id., The Collected Works, vol. X, Toronto, University of Toronto Press, p. 261-368.

PHELEP J.-Y., 1984, «Les critiques adressées par A. Comte et É. Durkheim à l'économie politique: le problème de l'abstraction», Thèse de $3^{\text {e }}$ cycle, Paris, Paris I Panthéon Sorbonne.

ROBBINS L., 2004 (1998), A History of Economic Thought: the LSE Lectures, Steven G Medema, Warren I Samuels et London school of economics and political science, Princeton (N.J.), Oxford University Press.

STEINER Ph., 1994, «Durkheim, les économistes et la critique de l'économie politique», Économies et Sociétés: cahiers de l'ISMEA, 28-4, p. 135-159.

-, 2005, L'École durkheimienne et l'économie: sociologie, religion et connaissance, Genève, Droz.

SWEDBERG R., 1994 (1987), Une histoire de la sociologie économique, Paris, Desclée De Brouwer.

VEBLEN Th., 1919 (1898), «Why is Economics not an Evolutionary Science?», in Id., The Place of Science in Modern Civilisation and Other Essays, B.W. Huebsch (éd.), p.56-81. 